\title{
Numerical quenching of a heat equation with nonlinear boundary conditions
}

\author{
Kouamé Béranger Edja ${ }^{\mathrm{a}, *}$, Kidjégbo Augustin Touréa ${ }^{\mathrm{a}}$ Brou Jean-Claude Koua ${ }^{\mathrm{b}}$ \\ a Institut National Polytechnique Félix Houphouët-Boigny Yamoussoukro, BP 2444, Côte d'Ivoire. \\ ${ }^{b}$ UFR Mathématique et Informatique, Université Félix Houphouët Boigny, Côte d'Ivoire.
}

\begin{abstract}
In this paper, we study the quenching behavior of semidiscretizations of the heat equation with nonlinear boundary conditions. We obtain some conditions under which the positive solution of the semidiscrete problem quenches in a finite time and estimate its semidiscrete quenching time. We also establish the convergence of the semidiscrete quenching time and obtain some results on numerical quenching rate. Finally we give some numerical results to illustrate our analysis.
\end{abstract}

Keywords: Numerical quenching, heat equation, nonlinear boundary.

2010 MSC: 35K05, 34B15, 74S20.

(C)2020 All rights reserved.

\section{Introduction}

In this paper, we study the behavior of a semidiscrete approximation of the following heat equation involving nonlinear boundary flux conditions:

$$
\begin{aligned}
u_{t}(x, t) & =u_{x x}(x, t), \quad x \in(0, a), t \in(0, T), \\
u_{x}(0, t) & =(1-u(0, t))^{-p}, u_{x}(a, t)=(1-u(a, t))^{-q}, t \in(0, T), \\
0 & \leqslant u(x, 0)=u_{0}(x)<a, x \in[0, a]
\end{aligned}
$$

where $m, n \geqslant 0, p, q>0$ and $u_{0}$ satisfies the compatibility conditions, i.e., $u_{0}^{\prime}(0)=\left(1-u_{0}(0)\right)^{-p}, u_{0}^{\prime}(a)=$ $\left(1-\mathfrak{u}_{0}(a)\right)^{-q}$. The problem is said to be quench if there exists a finite time T such that,

$$
\lim _{t \rightarrow T^{-}} \max _{0 \leqslant x \leqslant a} u(x, t)=1^{-} .
$$

The study of heat equation with nonlinear boundary conditions has deserved a great deal of interest in recent years and has been used to model, for example, heat transfer, polarization phenomena in ionic

\footnotetext{
${ }^{*}$ Corresponding author

Email addresses: kouame.edja@inphb.ci (Kouamé Béranger Edja), latoureci@gmail.com (Kidjégbo Augustin Touré),

k_brou@hotmail.com (Brou Jean-Claude Koua)

doi: $10.22436 /$ jnsa.013.01.06
}

Received: 2019-04-10 Revised: 2019-06-15 Accepted: 2019-06-16 
conductor, chemical catalyst kinetics, etc. (see $[3,4,6-9,14,15,17]$ and the references cited therein). In [4] the authors have given some conditions under which the solution $u$ of (1.1)-(1.3) quenches in a finite time and the quenching occurs only at the boundary $x=a$. They proved that $u_{t}$ blows up at quenching set and also gave the quenching rate near the quenching time $T$, which in other word says that there exist constants $C_{1}, C_{2}>0$ such that $1-C_{1}(T-t)^{\frac{1}{q+1}} \leqslant u(a, t) \leqslant 1-C_{2}(T-t)^{\frac{1}{q+1}}$.

From our knowledge, the numerical approximation of equations (1.1)-(1.3) has not been studied. Thus, in this paper, we are investigated in the numerical study using a semidiscrete form of (1.1)-(1.3). For that, we consider the case where $a=1$. Let $I \in \mathbb{N}$. We consider a uniform mesh on the interval $[0,1]$ as

$$
x_{i}=(i-1) h, i=1, \ldots, I, h=1 /(I-1),
$$

$\mathrm{U}_{\mathrm{h}}=\mathrm{U}_{\mathrm{h}}(\mathrm{t})=\left(\mathrm{U}_{1}(\mathrm{t}), \ldots, \mathrm{U}_{\mathrm{I}}(\mathrm{t})\right)^{\top}$ are the values of the numerical approximation at the nodes $x_{i}$ at time $t$ of the solution of (1.1)-(1.3). By the finite difference method we obtain the following system of ODEs whose solution is $\mathrm{U}_{\mathrm{h}}$ :

$$
\begin{aligned}
& u_{i}^{\prime}(t)=\delta^{2} u_{i}(t), \quad i=2, \ldots, I-1, t \in\left(0, T_{h}\right), \\
& u_{1}^{\prime}(t)=\delta^{2} u_{1}(t)-\frac{2\left(1-U_{1}(t)\right)^{-p}}{h}, t \in\left(0, T_{h}\right), \\
& u_{I}^{\prime}(t)=\delta^{2} u_{I}(t)+\frac{2\left(1-U_{I}(t)\right)^{-q}}{h}, t \in\left(0, T_{h}\right), \\
& u_{i}(0)=\varphi_{i} \geqslant 0, \quad i=1, \ldots, I,
\end{aligned}
$$

where $0 \leqslant \varphi_{i}<1,1 \leqslant i \leqslant I$ and

$$
\begin{aligned}
& \delta^{2} u_{i}(t)=\frac{u_{i-1}(t)-2 u_{i}(t)+U_{i+1}(t)}{h^{2}}, 2 \leqslant i \leqslant I-1, t>0, \\
& \delta^{2} u_{1}(t)=\frac{2 U_{2}(t)-2 u_{1}(t)}{h^{2}}, t>0, \quad \delta^{2} u_{I}(t)=\frac{2 U_{I-1}(t)-2 U_{I}(t)}{h^{2}}, t>0 .
\end{aligned}
$$

We say that the solution of (1.4)-(1.7) quenches in a finite time $T_{h}$ if

$$
\lim _{t \rightarrow T_{h}}\left\|U_{h}(t)\right\|_{\infty}=1
$$

$\mathrm{T}_{h}$ is called the semidiscrete quenching time of (1.4)-(1.7).

For study on numerical approximations of heat equation with non-linear boundary conditions we refer to $[1,5,10-13,16]$. Nabongo et al. in [12] were interested in the numerical study using a semidiscrete form of

$$
\begin{aligned}
& u_{t}(x, t)=u_{x x}(x, t), \quad x \in(0,1), t \in(0, T), \\
& u_{x}(0, t)=0, u_{x}(1, t)=-u(1, t)^{-\beta}, t \in(0, T), \quad u(x, 0)=u_{0}(x), x \in[0,1],
\end{aligned}
$$

where $\beta>0$. They showed that some conditions under which the positive solution of the numerical approximation for this heat equation quenches in a finite time, they also established the convergence of the semidiscrete quenching time and obtained some results on numerical quenching rate and set.

Inspired by $[4,5,12]$, we give some conditions under which any positive solution of semidiscrete scheme of (1.1)-(1.3) quenches in a finite time. We also show that the semidiscrete quenching time converges to the theoretical one when the mesh size goes to zero. The rest of the paper is organized as follows : in the next section, we give some properties concerning our semidiscrete scheme. In Section 3 , under some conditions, we prove that the solution of the semidiscrete scheme of (1.1)-(1.3) quenches in a finite time, we give a result on numerical quenching rate. In Section 4, we show that the quenching time converges to the theoretical one when the mesh size goes to zero. Finally, in the last section, we give some numerical results to illustrate our analysis. 


\section{Properties of the semidiscrete scheme}

In this section, we give some auxiliary results for the problem (1.4)-(1.7).

Definition 2.1. A function $V_{h} \in C^{1}\left(\left(0, T_{h}\right), \mathbf{R}^{I}\right)$ is a lower solution of (1.4)-(1.7) if

$$
\begin{aligned}
& V_{i}^{\prime}(t)-\delta^{2} V_{i}(t) \leqslant 0, \quad i=2, \ldots, I-1, t \in\left(0, T_{h}\right), \\
& V_{1}^{\prime}(t)-\delta^{2} V_{1}(t)+\frac{2\left(1-V_{1}(t)\right)^{-p}}{h} \leqslant 0, t \in\left(0, T_{h}\right), \\
& V_{I}^{\prime}(t)-\delta^{2} V_{I}(t)-\frac{2\left(1-V_{I}(t)\right)^{-q}}{h} \leqslant 0, t \in\left(0, T_{h}\right), \\
& V_{i}(0) \leqslant U_{i}(0), i=1, \ldots, I,
\end{aligned}
$$

where $U_{h}=\left(U_{1}, \ldots, U_{I}\right)^{\top}$ is solution of (1.4)-(1.7). On the other hand, we say that $V_{h} \in C^{1}\left(\left(0, T_{h}\right), \mathbf{R}^{I}\right)$ is an upper solution of (1.4)-(1.7) if these inequalities are reversed.

Lemma 2.2. Let $\mathrm{b}_{\mathrm{h}} \in \mathrm{C}^{0}\left(\left[0, \mathrm{~T}_{\mathrm{h}}\right), \mathbf{R}^{\mathrm{I}}\right)$ and let $\mathrm{V}_{\mathrm{h}} \in \mathrm{C}^{1}\left(\left[0, \mathrm{~T}_{\mathrm{h}}\right), \mathbf{R}^{\mathrm{I}}\right)$ such that

$$
\frac{d V_{i}(t)}{d t}-\delta^{2} V_{i}(t)+b_{i}(t) V_{i}(t) \geqslant 0,1 \leqslant i \leqslant I, t \in\left(0, T_{h}\right), \quad V_{i}(0) \geqslant 0,1 \leqslant i \leqslant I .
$$

Then we have $\mathrm{V}_{\mathrm{i}}(\mathrm{t}) \geqslant 0,1 \leqslant i \leqslant \mathrm{I}, \mathrm{t} \in\left(0, \mathrm{~T}_{\mathrm{h}}\right)$.

Proof. Let us define the vector $Z_{h}(t)=V_{h}(t) e^{\lambda t}$ with $\lambda$ a real. We have

$$
\begin{aligned}
Z_{i}^{\prime}(t)-\delta^{2} Z_{i}(t)+\left(b_{i}(t)-\lambda\right) Z_{i}(t) \geqslant 0, & i=1, \ldots, I, t \in\left(0, T_{h}\right), \\
Z_{i}(0) \geqslant 0, & i=1, \ldots, I .
\end{aligned}
$$

Denote $m=\min _{1 \leqslant i \leqslant I, t \in\left[0, T_{0}\right]} Z_{i}(t)$, where $T_{0} \in\left(0, T_{h}\right)$. Since for $i \in\{1, \ldots, I\}, Z_{i}(t)$ is a continuous function on the compact $\left[0, T_{0}\right]$, there exists $i_{0} \in\{1, \ldots, I\}$ such that $m=Z_{i_{0}}\left(t_{i_{0}}\right)$. Assume $m<0$. Taking $\lambda$ such that $b_{i_{0}}\left(t_{i_{0}}\right)-\lambda>0$. If $t_{i_{0}}=0$, then $Z_{i_{0}}(0)<0$, which contradicts (2.2), hence $t_{i_{0}}>0$.

It is not hard to see that

$$
Z_{i_{0}}^{\prime}\left(t_{i_{0}}\right)=\lim _{k \rightarrow 0} \frac{Z_{i_{0}}\left(t_{i_{0}}\right)-Z_{i_{0}}\left(t_{i_{0}}-k\right)}{k} \leqslant 0,
$$

moreover by a straightforward computation we get

$$
Z_{i_{0}}^{\prime}\left(t_{i_{0}}\right)-\delta^{2} Z_{i_{0}}\left(t_{i_{0}}\right)+\left(b_{i_{0}}\left(t_{i_{0}}\right)-\lambda\right) Z_{i_{0}}\left(t_{i_{0}}\right)<0,
$$

but these inequalities contradict (2.1) and the proof is complete.

Another form of the maximum principle for semidiscrete equations is the following comparison lemma.

Lemma 2.3. Let $W_{h}, V_{h} \in C^{1}\left(\left(0, T_{h}\right), \mathbf{R}^{I}\right)$ such that for $\mathrm{t} \in\left(0, T_{h}\right)$, we have

$$
\begin{aligned}
W_{i}^{\prime}(t)-\delta^{2} W_{i}(t) & \leqslant V_{i}^{\prime}(t)-\delta^{2} V_{i}(t), i=2, \ldots, I-1, \\
W_{1}^{\prime}(t)-\delta^{2} W_{1}(t)+g\left(W_{1}(t)\right) & \leqslant V_{1}^{\prime}(t)-\delta^{2} V_{1}(t)+g\left(V_{1}(t)\right), \\
W_{I}^{\prime}(t)-\delta^{2} W_{I}(t)+f\left(W_{1}(t)\right) & \leqslant V_{I}^{\prime}(t)-\delta^{2} V_{I}(t)+f\left(W_{I}(t)\right), \\
0 & \leqslant W_{i}(0) \leqslant V_{i}(0), i=1, \ldots, I .
\end{aligned}
$$

Then we have

$$
W_{h}(t) \leqslant V_{h}(t), \forall t \in\left(0, T_{h}\right)
$$


Proof. Let us define the vector $Z_{h}(t)=V_{h}(t)-W_{h}(t)$ with $\lambda$ a real. We have

$$
\begin{aligned}
Z_{i}^{\prime}(t)-\delta^{2} Z_{i}(t) & \geqslant 0, \quad i=2, \ldots, I-1, t \in\left(0, T_{h}\right), \\
Z_{1}^{\prime}(t)-\delta^{2} Z_{1}(t)+g^{\prime}(\zeta(t)) Z_{1}(t) & \geqslant 0, t \in\left(0, T_{h}\right), \\
Z_{I-1}^{\prime}(t)-\delta^{2} Z_{I-1}(t)-f^{\prime}(\xi(t)) Z_{I}(t) & \geqslant 0, t \in\left(0, T_{h}\right), \\
Z_{i}(0) & \geqslant 0, \quad i=1, \ldots, I,
\end{aligned}
$$

where $\zeta(t)$ and $\xi(t)$ lie, respectively, between $V_{1}(t)$ and $W_{1}(t)$, and between $V_{I}(t)$ and $W_{I}(t)$. We can rewrite (2.3)-(2.6) as

$$
Z_{i}^{\prime}(t)-\delta^{2} Z_{i}(t)+b_{i}(t) Z_{i}(t) \geqslant 0, \quad i=1, \ldots, I, t \in\left(0, T_{h}\right),
$$

where $b_{1}(t)=g^{\prime}(\zeta(t)), b_{I}(t)=-f^{\prime}(\xi(t))$ and $b_{i}(t)=0, i=2, \ldots, I-1, \forall t \in\left(0, T_{h}\right)$. According to lemma 2.2, $Z_{h}(t) \geqslant 0, \forall t \in\left(0, T_{h}\right)$ and the proof is complete.

The results of the next lemma are analogue to those of continuous problem.

Lemma 2.4. Let $\mathrm{U}_{\mathrm{h}} \in \mathrm{C}^{1}\left(\left(0, \mathrm{~T}_{\mathrm{h}}\right), \mathbf{R}^{\mathrm{I}}\right)$ be solution of (1.4)-(1.7) with a nonnegative initial data $\varphi_{\mathrm{h}}$ such that $\varphi_{\mathrm{h}}$ is a lower solution and $\varphi_{i+1}>\varphi_{i}$, for $i=1, \ldots, \mathrm{I}-1$. Then we have

(i) $\mathrm{U}_{\mathrm{i}}(\mathrm{t}) \geqslant 0$ and $\mathrm{U}_{\mathrm{i}}(\mathrm{t}) \geqslant \varphi_{i}, \quad \mathrm{i}=1, \ldots, \mathrm{I}, \mathrm{t} \in\left(0, \mathrm{~T}_{\mathrm{h}}\right)$;

(ii) $\mathrm{U}_{i+1}(\mathrm{t})>\mathrm{U}_{\mathrm{i}}(\mathrm{t}), i=1, \ldots, \mathrm{I}-1, \mathrm{t} \in\left(0, \mathrm{~T}_{\mathrm{h}}\right)$;

(iii) $\frac{d U_{i}(t)}{d t}>0, \quad i=1, \ldots, I, t \in\left(0, T_{h}\right)$.

Proof.

(i) Since $\varphi_{\mathrm{h}}$ is a lower solution of (1.4)-(1.7), we have

$$
\mathrm{U}_{\mathrm{i}}(\mathrm{t}) \geqslant \varphi_{\mathrm{i}} \geqslant 0, \quad \text { for } \quad \mathrm{i}=1, \ldots, \mathrm{I}, \mathrm{t} \in\left(0, \mathrm{~T}_{\mathrm{h}}\right),
$$

by Lemma 2.3 .

(ii) Let $t_{0}$ be the first $t>0$, such that $K_{i}(t)=U_{i+1}(t)-U_{i}(t)>0$, for $1 \leqslant i \leqslant I-1$, but $K_{i_{0}}\left(t_{0}\right)=$ $U_{i_{0}+1}\left(t_{0}\right)-U_{i_{0}}\left(t_{0}\right)=0$ for a certain $i_{0} \in\{1, \ldots, I-1\}$. Without lost of generality, we can suppose that $i_{0}$ is the smallest integer which satisfies the above equality. We have

$$
\begin{aligned}
\frac{d K_{i_{0}}\left(t_{0}\right)}{d t} & =\lim _{\epsilon \rightarrow 0} \frac{K_{i_{0}}\left(t_{0}\right)-K_{i_{0}}\left(t_{0}-\epsilon\right)}{\epsilon} \leqslant 0, \\
\frac{K_{i_{0}+1}\left(t_{0}\right)-2 K_{i_{0}}\left(t_{0}\right)+K_{i_{0}-1}\left(t_{0}\right)}{h^{2}}>0, & \text { if } 2 \leqslant i_{0} \leqslant I-2, \\
K_{i_{0}+1}\left(t_{0}\right)-3 K_{i_{0}}\left(t_{0}\right) h^{2}>0, & \text { if } i_{0}=1, \\
\frac{-3 K_{i_{0}}\left(t_{0}\right)+K_{i_{0}-1}\left(t_{0}\right)}{h^{2}}>0, & \text { if } i_{0}=I-1,
\end{aligned}
$$

which imply that

$$
\begin{array}{cl}
\frac{d K_{i_{0}}\left(t_{0}\right)}{d t}-\frac{K_{i_{0}+1}\left(t_{0}\right)-2 K_{i_{0}}\left(t_{0}\right)+K_{i_{0}-1}\left(t_{0}\right)}{h^{2}}<0, & \text { if } 2 \leqslant i_{0} \leqslant I-2, \\
\frac{d K_{i_{0}}\left(t_{0}\right)}{d t}-\frac{K_{i_{0}+1}\left(t_{0}\right)-3 K_{i_{0}}\left(t_{0}\right)}{h^{2}}-\frac{2\left(1-U_{i_{0}}(t)\right)^{-p}}{h}<0, & \text { if } i_{0}=1, \\
\frac{d K_{i_{0}}\left(t_{0}\right)}{d t}-\frac{-3 K_{i_{0}}\left(t_{0}\right)+K_{i_{0}-1}\left(t_{0}\right)}{h^{2}}-\frac{2\left(1-U_{i_{0}+1}(t)\right)^{-q}}{h}<0, & \text { if } i_{0}=I-1,
\end{array}
$$

but these inequalities contradict (1.4)-(1.6) and we obtain the desired result. 
(iii) Denote $Z_{i}(t)=U_{i}(t+\varepsilon)-U_{i}(t), \quad i=1, \ldots, I$, using (i) we obtain $Z_{i}(0) \geqslant 0$.

A straightforward calculation yields

$$
\begin{aligned}
\frac{d Z_{i}(t)}{d t} & =\frac{Z_{i-1}(t)-2 Z_{i}(t)+Z_{i+1}(t)}{h^{2}}, 2 \leqslant i \leqslant I-1, t \in\left(0, T_{h}\right), \\
\frac{d Z_{1}(t)}{d t} & =\frac{2 Z_{2}(t)-2 Z_{1}(t)}{h^{2}}-\frac{2 p(1-\eta(t))^{-p-1} Z_{1}(t)}{h}, t \in\left(0, T_{h}\right), \\
\frac{d Z_{I}(t)}{d t} & =\frac{2 Z_{I-1}(t)-2 Z_{I}(t)}{h^{2}}+\frac{2 q(1-\xi(t))^{-q-1} Z_{I}(t)}{h}, t \in\left(0, T_{h}\right), \\
Z_{i}(0) & \geqslant 0, \quad i=1, \ldots, I,
\end{aligned}
$$

where $\eta(t)$ and $\xi(t)$ lie, respectively, between $U_{1}(t+\varepsilon)$ and $U_{1}(t)$ and between $U_{I}(t+\varepsilon)$ and $U_{I}(t)$. We obtain the below inequality by the same manner as Lemma 2.3

$$
Z_{i}(t) \geqslant 0, \quad i=1, \ldots, I, \forall t \in\left(0, T_{h}\right) .
$$

This fact implies the desired result.

\section{Quenching and quenching rate}

In this section, under some assumptions, we show that the solution $U_{h}$ of (1.4)-(1.7) quenches in a finite time and estimate its semidiscrete quenching time. Moreover we determine the numerical quenching rate and quenching set of the solution.

Theorem 3.1. Let $\mathrm{U}_{\mathrm{h}}(\mathrm{t})$ be the solution of (1.4)-(1.7) such that the initial data $\varphi_{\mathrm{h}}$ is a lower solution, assumption $\mathrm{q} \geqslant \mathrm{p}>0$. Then there exists a positive constant $\gamma$ such that $\mathrm{U}_{\mathrm{h}}$ quenches in a finite time $\mathrm{T}_{\mathrm{h}}$ and we have the following estimate

$$
\mathrm{T}_{\mathrm{h}} \leqslant \frac{\left(1-\left\|\varphi_{\mathrm{h}}\right\|_{\infty}\right)^{\mathrm{q}+1}}{\gamma(\mathrm{q}+1)}
$$

Proof. Let $\left(0, T_{h}\right)$ be the maximal time interval on which $0 \leqslant U_{h}(t)<1$. Our aim is to show that $T_{h}$ is finite and satisfies the above inequality. Introduce the function $\mathrm{J}_{h}(t)$ such that

$$
J_{i}(t)=\frac{d U_{i}(t)}{d t}-\gamma\left(1-U_{i}(t)\right)^{-q}, i=1 \ldots, I .
$$

A straightforward calculation gives

$$
\begin{aligned}
\frac{d J_{1}(t)}{d t}-\delta^{2} J_{1}(t)+\frac{2 p}{h}\left(1-U_{1}(t)\right)^{-p-1} J_{1}(t) & =\frac{2 \gamma}{h}(q-p)\left(1-U_{1}(t)\right)^{-p-q-1}+\gamma\left(\delta^{+} u_{1}(t)\right)^{2} g^{\prime \prime}\left(\mu_{1}(t)\right), \\
\frac{d J_{I}(t)}{d t}-\delta^{2} J_{I}(t)-\frac{2 q}{h}\left(1-U_{I}(t)\right)^{-q-1} J_{I}(t) & =\gamma\left(\delta^{-} U_{I}(t)\right)^{2} g^{\prime \prime}\left(\theta_{I}(t)\right), \\
\frac{d J_{i}(t)}{d t}-\delta^{2} J_{i}(t) & =\gamma\left(\delta^{+} u_{i}(t)\right)^{2} g^{\prime \prime}\left(\mu_{i}(t)\right)+\gamma\left(\delta^{-} u_{i}(t)\right)^{2} g^{\prime \prime}\left(\theta_{i}(t)\right) i=2, \ldots, I-1,
\end{aligned}
$$

with $g^{\prime \prime}(x)=q(q+1)(1-x)^{-q-2}, \delta^{+} u_{i}=\frac{u_{i+1}-u_{i}}{h}, \delta^{-} u_{i}=\frac{u_{i}-u_{i-1}}{h}$, where $\theta_{i}$ and $\mu_{i}$ lie, respectively, between $U_{i}$ and $U_{i+1}$, and between $U_{i}$ and $U_{i-1}$. It is not hard to see that

$$
\begin{aligned}
& \frac{d J_{1}(t)}{d t}-\delta^{2} J_{1}(t)+\frac{2 p}{h}\left(1-U_{1}(t)\right)^{-p-1} J_{1}(t) \geqslant 0, \\
& \frac{d J_{I}(t)}{d t}-\delta^{2} J_{I}(t)-\frac{2 q}{h}\left(1-U_{I}(t)\right)^{-q-1} J_{I}(t) \geqslant 0, \\
& \frac{d J_{i}(t)}{d t}-\delta^{2} J_{i}(t) \geqslant 0, i=2, \ldots, I-1 \text {. }
\end{aligned}
$$


Moreover, using the fact that $\varphi_{h}$ is a lower solution, we obtain $J_{h}(0) \geqslant 0$. We deduce from Lemma 2.2 that $J_{i}(t) \geqslant 0, i=1, \ldots, I$. As a consequence we get

$$
\left(1-U_{i}(t)\right)^{q} d U_{i}(t) \geqslant \gamma d t .
$$

Integrating (3.1) from 0 to $t$ we obtain

$$
\left(1-\mathrm{U}_{i}(\mathrm{t})\right)^{\mathrm{q}+1} \leqslant\left(1-\varphi_{i}\right)^{\mathrm{q}+1}-\gamma(\mathrm{q}+1) \mathrm{t}, \quad i=1, \ldots, \mathrm{I} .
$$

Denote $\left\|\varphi_{h}\right\|_{\infty}=\varphi_{i_{0}}$ and $\left\|U_{h}(t)\right\|_{\infty}=U_{i_{0}}(t)$ for a certain $i_{0} \in\{1, \ldots, I\}$. Which means that $\left\|U_{h}\left(T_{0}\right)\right\|_{\infty}=1$ for some $T_{0}=\frac{\left(1-\left\|\varphi_{h}\right\|_{\infty}\right)^{q+1}}{\gamma(q+1)}$. Thus $U_{h}$ quenches in finite time $T_{h} \leqslant T_{0}$.

Theorem 3.2. Under the assumptions of Theorem 3.1 and Lemma 2.4, the solution $\mathrm{U}_{\mathrm{h}}$ of (1.4)-(1.7) quenches in a finite time $T_{h}$ and

$$
1-\left\|\mathrm{U}_{\mathrm{h}}(\mathrm{t})\right\|_{\infty} \sim\left(\mathrm{T}_{\mathrm{h}}-\mathrm{t}\right)^{\frac{1}{\mathrm{q}+1}} \text { for } \mathrm{t} \in\left(0, \mathrm{~T}_{\mathrm{h}}\right) .
$$

Proof. From Theorem $3.1 \mathrm{U}_{h}$ quenches in a finite time $T_{h}$. Integrating the above inequalities (3.1) over $\left(t, T_{h}\right)$ we obtain

$$
\mathrm{T}_{\mathrm{h}}-\mathrm{t} \leqslant \frac{\left(1-\mathrm{U}_{\mathrm{i}}(\mathrm{t})\right)^{\mathrm{q}+1}}{\gamma(\mathrm{q}+1)}, \quad i=1, \ldots, \mathrm{I},
$$

which implies that

$$
\left\|\mathrm{U}_{\mathrm{h}}(\mathrm{t})\right\|_{\infty} \leqslant 1-\mathrm{C}_{2}\left(\mathrm{~T}_{\mathrm{h}}-\mathrm{t}\right)^{1 /(\mathrm{q}+1)},
$$

where $C_{2}=(\gamma(q+1))^{1 /(q+1)}$. From Lemma $2.4, U_{i}<U_{i+1}$, for $1 \leqslant i \leqslant I-1$, hence $\left\|U_{h}(t)\right\|_{\infty}=U_{I}(t)$, for $t \in\left(0, T_{h}\right)$. It follows from (1.6) that

$$
\frac{d U_{I}(t)}{d t} \leqslant \frac{2}{h}\left(1-U_{I}(t)\right)^{-q}
$$

which implies that $\left(1-\mathrm{U}_{\mathrm{I}}(\mathrm{t})\right)^{\mathrm{q}} \mathrm{d} \mathrm{U}_{\mathrm{I}}(\mathrm{t}) \leqslant \frac{2}{\mathrm{~h}} \mathrm{dt}$. Thus we have

$$
1-C_{1}\left(T_{h}-t\right)^{1 /(q+1)} \leqslant\left\|U_{h}(t)\right\|_{\infty}, \text { where } C_{1}=\left(\frac{2(q+1)}{h}\right)^{1 /(q+1)},
$$

and we have the desired result.

Theorem 3.3. If $\lim _{\mathrm{t} \rightarrow \mathrm{T}_{\mathrm{h}}} \mathrm{U}_{\mathrm{I}}(\mathrm{t})=1$, then $\mathrm{U}_{\mathrm{h}}^{\prime}(\mathrm{t})$ blows up.

Proof. Suppose $U_{h}^{\prime}(t)$ is bounded. Then, there exists a positive constant $M$ such that $U_{h}^{\prime}(t)<M$. We have

$$
\sum_{i=2}^{I} \sum_{j=i}^{I} h^{2} u_{j}^{\prime}(t) \leqslant \sum_{i=2}^{I} \sum_{j=i}^{I} h^{2} M .
$$

From (1.4)-(1.6) and lemma 2.4 we get

$$
\frac{1}{\left(1-\mathrm{U}_{\mathrm{I}}(\mathrm{t})\right)^{\mathrm{q}}}<\frac{\mathrm{M}}{2}+\frac{\mathrm{hM}}{2}+\mathrm{U}_{\mathrm{I}}(\mathrm{t})-\mathrm{U}_{1}(\mathrm{t})
$$

As $t \rightarrow T_{h}^{-}$, the left-hand side tends to infinity while the right-side is finite. This contradiction shows that $\mathrm{u}_{\mathrm{h}}^{\prime}(\mathrm{t})$ blows up. 


\section{Convergence of the semidiscrete quenching time}

In this section, we study the convergence of the semidiscrete quenching time. Now we will show that for each fixed time interval $[0, T]$, where $u$ is defined, the solution $U_{h}$ of (1.4)-(1.7) approximates $u$ when the mesh parameter $h$ goes to zero. We denote

$$
u_{h}(t)=\left(u\left(x_{1}, t\right), \ldots, u\left(x_{I}, t\right)\right)^{\top} \text { and }\left\|u_{h}(t)\right\|_{\infty}=\max _{1 \leqslant i \leqslant I}\left|u_{i}(t)\right| .
$$

Theorem 4.1. Assume that the problem (1.1)-(1.3) has a solution $\mathrm{u} \in \mathrm{C}^{4,1}\left([0,1] \times\left[0, \mathrm{~T}^{*}\right]\right)$ and the initial condition $\varphi_{\mathrm{h}}$ at (1.7) verifies

$$
\left\|\varphi_{\mathrm{h}}-\mathrm{u}_{\mathrm{h}}(0)\right\|_{\infty}=\mathrm{o}(1), \mathrm{h} \rightarrow 0 .
$$

Then, for $\mathrm{h}$ small enough, the semidiscrete problem (1.4)-(1.7) has a unique solution $\mathrm{U}_{\mathrm{h}} \in \mathrm{C}^{1}\left(\left[0, \mathrm{~T}^{*}\right], \mathbf{R}^{\mathrm{I}}\right)$ such that

$$
\max _{t \in\left[0, T^{*}\right]}\left\|\mathrm{U}_{\mathrm{h}}(\mathrm{t})-\mathrm{u}_{\mathrm{h}}(\mathrm{t})\right\|_{\infty}=\mathrm{O}\left(\left\|\varphi_{\mathrm{h}}-\mathrm{u}_{\mathrm{h}}(0)\right\|_{\infty}+\mathrm{h}^{2}\right) \text {, as } \mathrm{h} \rightarrow 0 .
$$

Proof. Let $\sigma>0$ be such that

$$
\|\mathrm{u}\|_{\infty}<\sigma, \mathrm{t} \in\left[0, \mathrm{~T}^{*}\right] .
$$

Then the problem (1.4)-(1.7), for each $h$, has a unique solution $U_{h} \in C^{1}\left(\left[0, T^{*}\right], \mathbf{R}^{I}\right)$. Let $t(h) \leqslant T^{*}$ be the greatest value of $t>0$ such that

$$
\left\|\mathrm{U}_{\mathrm{h}}(\mathrm{t})-\mathrm{u}_{\mathrm{h}}(\mathrm{t})\right\|_{\infty}<1
$$

The relation (4.1) implies $t(h)>0$ for $h$ small enough. Using the triangle inequality, we obtain

$$
\left\|U_{h}(t)\right\|_{\infty} \leqslant\left\|U_{h}(t)-u_{h}(t)\right\|_{\infty}+\|u(\cdot, t)\|_{\infty}, \text { for } t \in(0, t(h)),
$$

which implies that

$$
\left\|\mathrm{U}_{\mathrm{h}}(\mathrm{t})\right\|_{\infty} \leqslant 1+\sigma, \text { for } \mathrm{t} \in(0, \mathrm{t}(\mathrm{h})) .
$$

Let $e_{h}(t)=U_{h}(t)-u_{h}(t), \forall t \in\left[0, T^{*}\right]$ be the discretization error and let $W \in C^{4,1}\left([0,1],\left[0, T^{*}\right]\right)$ be such that $W(x, t)=\left(\left\|\varphi_{h}-u_{h}(0)\right\|_{\infty}+M h^{2}\right) e^{(M+1) t+\sqrt{M+1} x}, \forall(x, t) \in[0,1] \times\left[0, T^{*}\right]$, with $M$ a positive constant. We can prove by Lemma 2.3 that

$$
\left|e_{i}(t)\right|<W\left(x_{i}, t\right), 1 \leqslant i \leqslant I, \text { for } t \in(0, t(h)) .
$$

We deduce that

$$
\left\|\mathrm{U}_{h}(\mathrm{t})-\mathrm{u}_{\mathrm{h}}(\mathrm{t})\right\|_{\infty} \leqslant\left(\left\|\varphi_{h}-u_{h}(0)\right\|_{\infty}+M h^{2}\right) e^{(M+1) t+\sqrt{M+1}}, \text { for } t \in(0, t(h)) .
$$

Suppose that $T^{*}>t(h)$ from (4.2), we obtain

$$
1=\left\|\mathrm{U}_{\mathrm{h}}(\mathfrak{t}(\mathrm{h}))-\mathfrak{u}_{\mathrm{h}}(\mathrm{t}(\mathrm{h}))\right\|_{\infty} \leqslant\left(\left\|\varphi_{\mathrm{h}}-\mathfrak{u}_{\mathrm{h}}(0)\right\|_{\infty}+\mathrm{Mh}^{2}\right) \mathrm{e}^{(M+1) t+\sqrt{M+1}} .
$$

Since the term on the right hand side of the above inequality goes to zero as $h$ tends to zero, we deduce that, $1 \leqslant 0$, which is impossible. Hence we have $t(h)=T^{*}$, and the proof is complete.

Theorem 4.2. Suppose that the solution $\mathrm{u}$ of (1.1)-(1.3) quenches in a finite time $\mathrm{T}$ such that $\mathrm{u} \in \mathrm{C}^{4,1}([0,1] \times[0, \mathrm{~T}))$ and the initial condition at (1.7) satisfies

$$
\left\|\varphi_{\mathrm{h}}-\mathrm{u}_{\mathrm{h}}(0)\right\|_{\infty}=\mathrm{o}(1), \mathrm{h} \rightarrow 0 .
$$

Then the solution $\mathrm{U}_{\mathrm{h}}$ of (1.4)-(1.7) quenches in a finite time $\mathrm{T}_{\mathrm{h}}$ and we have

$$
\lim _{h \rightarrow 0} T_{h}=T \text {. }
$$


Proof. Set $\varepsilon>0$. There exists $\eta>0$ such that

$$
\frac{(1-y)^{q+1}}{\gamma(q+1)} \leqslant \frac{\varepsilon}{2}, \quad 0 \leqslant y \leqslant \eta
$$

Since $u(x, t)$ quenches in a finite time $T$, there exists a time $T_{0}<T$ such that $\left|T_{0}-T\right|<\frac{\varepsilon}{2}$ and $0 \leqslant$ $\|\left. u(x, t)\right|_{\infty} \leqslant \frac{\eta}{2}$ for $t \in\left[T_{0}, T\right)$. Setting $T_{1}=\frac{T_{0}+T}{2}$, it is not hard to see that $\|\left. u(x, t)\right|_{\infty}<1$ for $t \in\left[0, T_{1}\right]$. From Theorem 4.1, we have $\left\|\mathrm{U}_{h}\left(\mathrm{~T}_{1}\right)-\mathrm{u}_{\mathrm{h}}\left(\mathrm{T}_{1}\right)\right\|_{\infty} \leqslant \frac{\eta}{2}$. Applying the triangle inequality, we get

$$
\left\|\left.\mathrm{U}_{\mathrm{h}}\left(\mathrm{T}_{1}\right)\right|_{\infty} \leqslant\right\| \mathrm{U}_{\mathrm{h}}\left(\mathrm{T}_{1}\right)-\mathrm{u}_{\mathrm{h}}\left(\mathrm{T}_{1}\right)\left\|_{\infty}+\right\| \mathrm{u}_{\mathrm{h}}\left(\mathrm{T}_{1}\right) \|_{\infty} \leqslant \eta .
$$

From Theorem 3.1, $\mathrm{U}_{h}$ quenches in a finite time $T_{h}$. We deduce from (3.2) and (4.3) that

$$
\left|T_{h}-T\right| \leqslant\left|T_{h}-T_{1}\right|+\left|T_{1}-T\right| \leqslant \frac{\left(1-\|\left. U_{h}\left(T_{1}\right)\right|_{\infty}\right)^{q+1}}{\gamma(q+1)}+\frac{\varepsilon}{2} \leqslant \varepsilon .
$$

\section{Numerical experiments}

In this section, we present some numerical approximations to the quenching time of (1.4)-(1.7), the initial data $\varphi_{\mathrm{h}}$ such

$$
\alpha \varphi_{2} \geqslant\left(1-\varphi_{1}\right)^{-p},
$$

where $\alpha>0$. We use the following explicit scheme

$$
\begin{aligned}
\frac{U_{i}^{(n+1)}-U_{i}^{(n)}}{\Delta t_{h}^{n e}} & =\frac{U_{i+1}^{(n)}-2 U_{i}^{(n)}+U_{i-1}^{(n)}}{h^{2}}, \quad 2 \leqslant i \leqslant I-1, \\
\frac{U_{1}^{(n+1)}-U_{1}^{(n)}}{\Delta t_{h}^{n e}} & =\frac{2 U_{2}^{(n)}-2 U_{1}^{(n)}}{h^{2}}-\frac{2}{h}\left(1-U_{1}^{(n)}\right)^{-p}, \\
\frac{U_{I}^{(n+1)}-U_{I}^{(n)}}{\Delta t_{h}^{n e}} & =\frac{2 U_{I-1}^{(n)}-2 U_{I}^{(n)}}{h^{2}}+\frac{2}{h}\left(1-U_{I}^{(n)}\right)^{-q}, \\
U_{i}^{(0)} & =\varphi_{i}, \quad 1 \leqslant i \leqslant I,
\end{aligned}
$$

where $n \geqslant 0, \Delta t_{h}^{n e}=\min \left\{\frac{h^{2}}{2}, h^{2}\left\|U_{h}^{(n)}\right\|_{\infty}^{q+1}\right\}$. We also consider the implicit scheme

$$
\begin{aligned}
\frac{u_{i}^{(n+1)}-u_{i}^{(n)}}{\Delta t_{h}^{n}} & =\frac{u_{i+1}^{(n+1)}-2 U_{i}^{(n+1)}+U_{i-1}^{(n+1)}}{h^{2}}, \quad 2 \leqslant i \leqslant I-1, \\
\frac{u_{1}^{(n+1)}-U_{1}^{(n)}}{\Delta t_{h}^{n}} & =\frac{2 U_{2}^{(n+1)}-2 U_{1}^{(n+1)}}{h^{2}}-\frac{2}{h}\left(1-u_{1}^{(n)}\right)^{-p}, \\
\frac{u_{I}^{(n+1)}-U_{I}^{(n)}}{\Delta t_{h}^{n}} & =\frac{2 U_{I-1}^{(n+1)}-2 U_{I}^{(n+1)}}{h^{2}}+\frac{2}{h}\left(1-U_{I}^{(n)}\right)^{-q}, \\
u_{i}^{(0)} & =\varphi_{i}, \quad 1 \leqslant i \leqslant I,
\end{aligned}
$$

where $n \geqslant 0, \Delta t_{h}^{n}=h^{2}\left\|U_{h}^{(n)}\right\|_{\infty}^{q+1}$.

Let us notice that (5.1) and the restriction on the time step guarantee the positivity of the discrete solution. We need the following definition.

Definition 5.1. We say that the discrete solution $u_{h}^{(n)}$ of the explicit scheme or the implicit scheme quenches in a finite time if $\lim _{n \rightarrow \infty}\left\|\mathrm{U}_{h}^{(n)}\right\|_{\infty}=1$ and the series $\sum_{n=0}^{+\infty} \Delta t_{h}^{n}$ converges. The quantity $\mathrm{t}_{\mathrm{h}}^{\mathrm{n}}=\sum_{j=0}^{n-1} \Delta \mathrm{t}_{\mathrm{h}}^{j}$ is called the numerical quenching time of the solution $\mathrm{U}_{h}^{(\mathfrak{n})}$ and $\mathrm{T}_{\mathrm{h}}=\sum_{\mathfrak{n}=0}^{+\infty} \Delta \mathrm{t}_{\mathrm{h}}^{n}$ is called the numerical quenching time of the solution $\mathrm{U}_{h}$. 
In Tables 1, 2, 3, and 4, in rows, we present the numerical quenching times, the numbers of iterations and the orders of the approximations corresponding to meshes of $16,32,64,128,256,512,1024$. We take for the numerical quenching time $T_{h}=\sum_{n=0}^{+\infty} \Delta t_{h}^{n}$ which is computed at the first time when $\Delta t_{h}^{n}=$ $\left|t_{h}^{n+1}-t_{h}^{n}\right| \leqslant 10^{-16}$. The order(s) of the method is computed from

$$
s=\frac{\log \left(\left(T_{4 h}-T_{2 h}\right) /\left(T_{2 h}-T_{h}\right)\right)}{\log (2)}, \text { where } h=1 /(I-1) .
$$

For the numerical values, we take $\varphi_{i}=\sqrt{1.5}-\cos \left(\frac{\Pi(i-1) h}{4}\right)$ for $i=1, \ldots, I$.

Table 1: Numerical quenching times obtained with the explicit Euler method for $\mathrm{q}=0.5, \mathrm{p}=0.5$.

\begin{tabular}{|c|c|c|c|}
\hline $\mathrm{I}$ & $\mathrm{T}_{\mathrm{h}}$ & $\mathrm{n}$ & $\mathrm{s}$ \\
\hline 16 & 0.04139596 & 236 & - \\
\hline 32 & 0.03955588 & 679 & - \\
\hline 64 & 0.03900581 & 2164 & 1.74 \\
\hline 128 & 0.03884205 & 7556 & 1.75 \\
\hline 256 & 0.03879396 & 28153 & 1.77 \\
\hline 512 & 0.03878005 & 108750 & 1.79 \\
\hline 1024 & 0.03877610 & 427914 & 1.82 \\
\hline
\end{tabular}

Table 3: Numerical quenching times, obtained with the explicit Euler method for $\mathrm{q}=1, \mathrm{p}=0.5$.

\begin{tabular}{|c|c|c|c|}
\hline $\mathrm{I}$ & $\mathrm{T}_{\mathrm{h}}$ & $\mathrm{n}$ & $\mathrm{s}$ \\
\hline 16 & 0.00972448 & 129 & - \\
\hline 32 & 0.00813955 & 310 & - \\
\hline 64 & 0.00763971 & 816 & 1.66 \\
\hline 128 & 0.00748449 & 2434 & 1.69 \\
\hline 256 & 0.00743743 & 8155 & 1.72 \\
\hline 512 & 0.00742349 & 29675 & 1.76 \\
\hline 1024 & 0.00741945 & 113290 & 1.79 \\
\hline
\end{tabular}

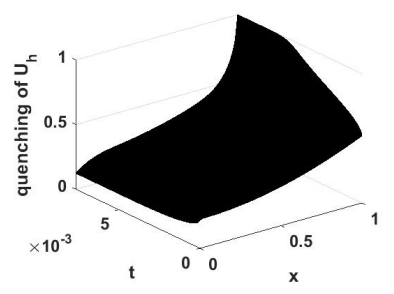

Figure 1: Evolution of the numerical solution $\mathrm{U}_{\mathrm{h}}$ for $\mathrm{I}=$ $64, \mathrm{q}=1$ and $\mathrm{p}=0.5$.
Table 2: Numerical quenching times obtained with the implicit Euler method for $q=0.5, p=0.5$.

\begin{tabular}{|c|c|c|c|}
\hline $\mathrm{I}$ & $\mathrm{T}_{\mathrm{h}}$ & $\mathrm{n}$ & $\mathrm{s}$ \\
\hline 16 & 0.04221929 & 237 & - \\
\hline 32 & 0.03975759 & 680 & - \\
\hline 64 & 0.03905612 & 2163 & 1.81 \\
\hline 128 & 0.03885465 & 7558 & 1.80 \\
\hline 256 & 0.03879711 & 28155 & 1.81 \\
\hline 512 & 0.03878084 & 108753 & 1.82 \\
\hline 1024 & 0.03877629 & 427917 & 1.84 \\
\hline
\end{tabular}

Table 4: Numerical quenching times obtained with the implicit Euler method for $q=1, p=0.5$.

\begin{tabular}{|c|c|c|c|}
\hline $\mathrm{I}$ & $\mathrm{T}_{\mathrm{h}}$ & $\mathrm{n}$ & $\mathrm{s}$ \\
\hline 16 & 0.01036564 & 129 & - \\
\hline 32 & 0.00830903 & 311 & - \\
\hline 64 & 0.00768367 & 818 & 1.72 \\
\hline 128 & 0.00749576 & 2436 & 1.73 \\
\hline 256 & 0.00744028 & 8158 & 1.76 \\
\hline 512 & 0.00742421 & 29678 & 1.79 \\
\hline 1024 & 0.00741963 & 113293 & 1.81 \\
\hline
\end{tabular}

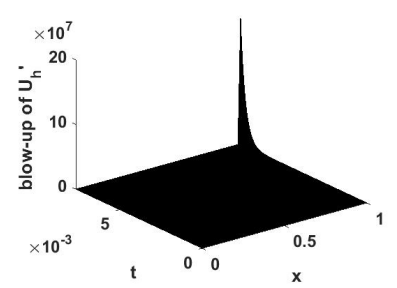

Figure 2: Evolution of $U_{h}^{\prime}$ for $I=64, q=1$ and $p=0.5$.

Remark 5.2. The various tables of our numerical results show that there is a relationship between the quenching time and the flows on the boundaries. If we consider the problem (1.4)-(1.7) in the case where the initial data $\varphi(x)=\sqrt{1.5}-\cos \left(\frac{\Pi x}{4}\right)$ and $q=0.5$, we observe from Tables 1 and 2 that the numerical quenching time is approximately equal to 0.038 . When $\mathrm{q}=1$, we observe from Tables 3 and 4 that the numerical blow-up time is approximately equal to 0.0074 . Thus we can said that when rise $q$ we have an acceleration of quench of the solution. Also, from the tables we observe the convergence of blow-up time $\mathrm{T}_{h}$ of the solution of (1.4)-(1.7), since the rate of convergence is near 2. This result does not surprise us because of the result established in the previous section. 


\section{References}

[1] K. A. Adou, K. A. Touré, A. Coulibaly, Numerical study of the blow-up time of positive solutions of semilinear heat equations, Far East J. Appl. Math., 4 (2018), 291-308. 1

[2] T. K. Boni, Extinction for discretizations of some semilinear parabolic equations, C. R. Acad. Sci. Paris Sér. I Math., 333 (2001), 795-800.

[3] T. K. Boni, B. Y. Diby, Quenching time of solutions for some nonlinear parabolic equations with Dirichlet boundary condition and a potential, Ann. Math. Inform., 35 (2008), 31-42. 1

[4] C. Y. Chan, S. I. Yuen, Parabolic problems with nonlinear absorptions and releases at the boundaries, Appl. Math. Comput., 121 (2001), 203-209. 1, 1

[5] K. B. Edja, K. A. Touré, B. J.-C. Koua, Numerical Blow-up for a Heat Equation with Nonlinear Boundary Conditions, J. Math. Res., 10 (2018), 119-128. 1

[6] H. Kawarada, On Solutions of Initial-Boundary Problem for $\mathfrak{u}_{\mathrm{t}}=\mathrm{u}_{\mathrm{x} x}+\frac{1}{1-\mathrm{u}}$, Publ. RIMS, Kyoto Univ., 10 (1975), 729-736. 1

[7] C. M. Krirk, C. A. Roberts, A quenching problem for the heat equation, J. Integral Equations Appl., 14 (2002), 53-72.

[8] H. A. Levine, The Quenching of Solutions of Linear Parabolic and Hyperbolic Equations with Nonlinear Boundary Conditions, SIAM J. Math. Anal., 4 (1983), 1139-1153.

[9] H. A. Levine, J. T. Montgomery, The quenching of solutions of some nonlinear parabolic equations, SIAM J. Math. Anal., 11 (1980), 842-847. 1

[10] K. W. Liang, P. Lin, R. C. E. Tan, Numerical Solution of Quenching Problems Using Mesh-Dependent Variable Temporal Steps, Appl. Numer. Math., 57 (2007), 791-800. 1

[11] T. M. Mathurin, T. K. Augustin, M. E. Patrice, Numerical approximation of the blow-up time for a semilinear parabolic equation with nonlinear boundary equation, Far East J. Math. Sci. (FJMS), 60 (2012), 125-167.

[12] D. Nabongo, T. K. Boni, Quenching for semidiscretizations of a heat equation with a singular boundary condition, Asymptot. Anal., 59 (2008), 27-38. 1

[13] K. C. N'dri, K. A. Touré, G. Yoro, Numerical blow-up time for a parabolic equation with nonlinear boundary conditions, Int. J. Numer. Methods Appl., 17 (2018), 141-160. 1

[14] B. Seluk, Quenching behavior of a semilinear reaction-diffusion system with singular boundary condition, Turkish J. Math., 40 (2016), 166-180. 1

[15] B. Seluk, N. Ozalp, The quenching behavior of a semilinear heat equation with a singular boundary outflux, Quart. Appl. Math., 72 (2014), 747-752. 1

[16] M. G. Teixeira, M. A. Rincon, I.-S. Liu, Numerical analysis of quenching-Heat conduction in metallic materials, Appl. Math. Model., 33 (2009), 2464-2473. 1

[17] Y. H. Zhi, C. L. Mu, The quenching behavior of a nonlinear parabolic equation with nonlinear boundary outflux, Appl. Math. Comput., 184 (2007), 624-630. 1 